\title{
Insights of Human Gut Microbiota
}

\author{
R. Sundaralingam \\ Assistant Professor, Department of Microbiology, \\ Madras Christian College (Autonomous), \\ Tambaram, Chennai, Tamil nadu, India.
}

\begin{abstract}
Are Microbes, Mostly Bacteria That Are Found on the Body of a Healthy Person?
A growing body of evidence highlights the importance of the gut microbiota in early life. With technical advancements, we are yet to uncover our natural history by accounting for the vast diversity of microbial life which colonizes the human body. The word microbiota represents an ensemble of microorganisms that resides in a previously established environment.
\end{abstract}

Gut microbiota (formerly called gut flora) is the name given today to the microbe population living in our intestine. One third of our gut microbiota is common to most people, while two thirds are specific to each one of us. In other words, the microbiota in our intestine is like an individual identity card.

Until recently, it was assumed that the fetal environment is sterile and that microbial colonization begins with birth. However, with the advent of culture-independent molecular methods, recent evidence has called this assumption into question. The placenta, amniotic fluid, fetal membranes, and cord blood from healthy, term pregnancies have been shown to harbor microorganisms, suggesting that the presence of bacteria in these tissues is not necessarily indicative of a pathogenic state.

The Human Gut Microbiota is a complex dynamic system with the potential for multistability. The Gut microbiota continuously changes from birth up to the most extreme limits of human life, reconfiguring its metagenomic layout in response to daily variations in diet or specific host physiological and immunological needs at different ages.

Some functions of gut microbiota include:

- It helps the body to digest certain foods that the stomach and small intestine have not been able to digest.

- It helps with the production of some vitamins (B and K).

- It helps us combat aggressions from other microorganisms, maintaining the wholeness of the intestinal mucosa.

- It plays an important role in the immune system, performing a barrier effect.

- A healthy and balanced gut microbiota is the key to ensure proper digestive functioning.

Considering the major role gut microbiota plays in the normal functioning of the body and the different functions it accomplishes, experts nowadays consider it as an "organ". The composition of our microbiota evolves throughout our entire life, from birth to old age, and is the result of different environmental influences. Imbalance in the composition of this bacterial community in the human gut can lead to transient intestinal dysfunctions and pathological states. This imbalance of the normal gut microbiota have been linked with gastrointestinal conditions such as inflammatory bowel disease (IBD) and irritable bowel syndrome (IBS), and wider systemic manifestations of disease such as obesity, type 2 diabetes, and atopy.

Taking into account that development of the Gut microbiota may begin before birth, we must explore the factors and exposures that influence this process. It is clear that the gut microbiota plays a major role in health and disease; one that can be influenced by external factors, making it an exciting focus 


\section{R. Sundaralingam}

for research. Our understanding of the composition and functions of the human gut microbiota is crucial to assess their metabolic potential and influence on human health.

Human studies are currently limited by practical difficulties associated with obtaining microbial samples from the gut. By addressing such challenges, researchers hope to gain a greater understanding of the impact of microbes on obesity and metabolic diseases, and may one day be able to offer personalized microbial therapies to target these and other health issues. 